\title{
Strom à la carte - Vielfalt der Anwendungen und Lösungen
}

\section{Fachtagung der Österreichischen Gesellschaft für Energietechnik im OVE}

J. Vavra

Online publiziert am 20. November 2017

(C) Springer-Verlag GmbH Austria, ein Teil von Springer Nature 2017

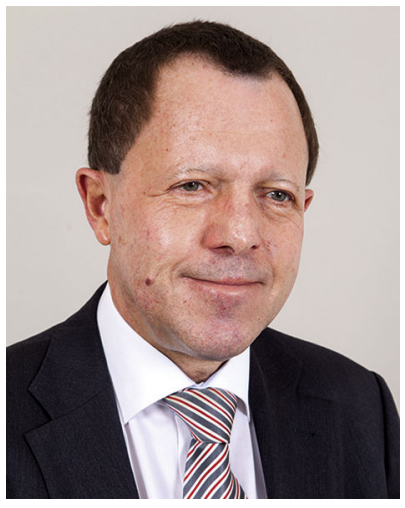

Prok. Dipl.-Ing. Johannes Vavra
Sehr geehrte Damen und Herren

ich begrüße Sie sehr herzlich zu unserer traditionellen Veranstaltung, nämlich zur 55. Fachtagung der Österreichischen Gesellschaft für Energietechnik im OVE, welche heuer in Salzburg stattfindet.

Neben dem gewissermaßen kleineren Jubiläum der 55. Fachtagung gilt es heuer übrigens auch, das 50-jährige Bestehen der OGE entsprechend zu würdigen. Doch wie kommt es, dass wir bereits die 55. Fachtagung im 50. Bestandsjahr der OGE begehen?

Im Jahr 1964 wurde an der Versuchs- und Forschungsanstalt für Hochspannungstechnik in Graz eine unabhängige Interessensgemeinschaft gebildet, deren Angehörige es sich zur Aufgabe machten, bei regelmäßig (gelegentlich auch öfter als ein Mal pro Jahr) stattfindenden Treffen "aktuelle Probleme auf dem Gebiet der Hochspannungstechnik in freiem Gedankenaustausch zu diskutieren". Aus diesen Fachgesprächen gingen die Fachtagungen hervor, und so sind wir heuer bereits bei der 55 . angelangt.

Als Gründungsjahr der Österreichischen Gesellschaft für Energietechnik gilt hingegen 1967, weil sich in diesem Jahr die Sektion "Hochspannungs- und Hochstromtechnik" im OVE konstituierte. 50 Jahre OGE daher, wenngleich die Bezeichnung OGE erst seit dem Jahr 1990 verwendet wird, als nämlich der Name der oben genannten Sektion auf "Österreichische Gesellschaft für Energietechnik" geändert wurde.

Doch zurück zur diesjährigen OGE-Fachtagung, für die wir den Titel "Strom à la carte - Vielfalt der Anwendungen und Lösungen" gewählt haben: Nach einem Rückblick auf die Entwicklung der Stromversorgung und Stromnutzung in den letzten 50 Jahren und einer aktuellen Betrachtung der Stromversorgung und -übertragung sowie Lastdeckung in Europa mit Fokus Österreich, kommen diesmal repräsentative Anwender zu Wort, um ihre spezifischen Anforderungen an die Stromversorgung zu beschreiben.
Darunter befinden sich Vertreter eines industriellen Technologieunternehmens und der Halbleiterindustrie, eines Rechenzentrums, eines Großkrankenhauses sowie des Bereiches Tourismus und Schilifte. Wie diesen Anforderungen heute und in Zukunft entsprochen werden kann, wird durch hochrangige Experten der Elektrizitätswirtschaft, der Elektroindustrie sowie aus Lehre und Forschung aufgezeigt, sodass ein spannender Dialog mit interessantem Erfahrungsaustausch erwartet werden darf.

Insgesamt gilt es auch heuer wieder, dem Anspruch einer Fachtagung gerecht zu werden und daher konkrete Antworten in Form von Konzepten oder bereits realisierten organisatorischen und technischen Lösungen zu bieten. Für diese "Vielfalt der Anwendungen und Lösungen" stehen an diesen beiden Tagen hochkarätige Referenten aus allen einschlägigen Bereichen zur Verfügung, um die Herausforderungen darzustellen und notwendige Maßnahmen zu beschreiben, Lösungsansätze vorzustellen, die Themen aus unterschiedlichen Blickwinkeln zu beleuchten und umfassend zu diskutieren.

Natürlich lade ich Sie, sehr geehrte Teilnehmerinnen und Teilnehmer, dazu ein, sich ebenfalls intensiv an diesen Diskussionen zu beteiligen, Ihre diesbezüglichen Erfahrungen einzubringen und darüber hinaus die vielfältigen Gelegenheiten zum Gedankenaustausch bei diesem Branchentreff zu nutzen.

Abschließend möchte ich darauf hinweisen, dass auch heuer wieder die Verleihung der Oesterreichs Energie-Preise, des Prof. Werner Rieder-Preises und der OGE-Preise im Rahmen der heutigen Abendveranstaltung erfolgen wird. Diese Veranstaltung, zu der ich Sie natürlich gleichfalls herzlich einladen darf, wird im historischen Ambiente des Hofbräu Kaltenhausen in Hallein stattfinden.

Ich wünsche somit allen Teilnehmerinnen, Teilnehmern und Referenten eine sehr abwechslungsreiche, informative sowie qualitativ hochwertige Veranstaltung und darf Sie auf der OGE-Fachtagung 2017 in Salzburg herzlich willkommen heißen!

Begrüßung bei der 55. Fachtagung der Österreichischen Gesellschaft für Energietechnik (OGE) im OVE, die am 12. und 13. Oktober 2017 in Salzburg stattfand.

Johannes Vavra Vorstandsvorsitzender der Österreichischen Gesellschaft für Energietechnik (OGE) im OVE, Stabsstellenleiter bei Wiener Netze GmbH.

Vavra, Johannes, Wiener Netze GmbH，Erdbergstraße 236, 1110 Wien, Österreich (E-Mail: johannes.vavra@wienernetze.at) 